\title{
Investigation on Fragmentation of Pyrophoric Alloy Samples during Taylor Test Using SPH
}

\author{
Evaristo Santamaria Ferraro ${ }^{1,2}$, Marina Seidl $^{1}$ and Tom De Vuyst ${ }^{2}$ \\ ${ }^{1}$ ISL - French German Research Institute of Saint Louis, 68300 Saint Louis, France \\ ${ }^{2}$ University of Hertfordshire, School of Computer Science and Engineering, AL109EU Hatfield, UK
}

\begin{abstract}
This study is the first part of a project that aims to assess and model impact-induced energy release (IIER). The present part of the work investigates the failure mode of brittle commercial pyrophoric alloy samples during Taylor impact tests. A series of ferrocerium specimens were shot against tungsten carbide anvils, with velocities ranging between 60 and 140 $\mathrm{m} / \mathrm{s}$. A Total Lagrangian SPH model was employed to simulate the deformation and impact-induced fragmentation of the cylinders using LSDYNA ${ }^{\circledR}$. The modified Johnson-Cook constitutive model was applied in combination with the Cockcroft-Latham fracture criterion. The plastic deformation process, shear cracking, and fragmentation are well reproduced in the numerical results.
\end{abstract}

\section{Introduction}

The work discussed in this paper is the first step of a broader project aiming to investigate and model Impact Induced Energy Release (IIER) of Intermetallic Reactive Materials (IRM). The improvement in material design and manufacturing drove the attention of defence industry towards IRM in recent years, as they show interesting mechanical properties combined with the capability to release energy when stimulated [1]. Furthermore, IRM are stable in normal conditions, allowing relatively safe handling. This preliminary study is performed using Ferrocerium, a commercially available brittle pyrophoric mixture of rare earths, mainly cerium (Ce, 49\%) and lanthanum (La, 23\%) [2], with mechanical properties comparable to common IRMs, which releases energy upon impact. Ferrocerium is used in this work as a surrogate material to set up the academic study and evaluate the methodologies necessary for data extrapolation and further progression of the research. In particular, this work focuses on a series of Taylor impact tests performed to examine the failure mode and its evolution for Ferrocerium bars.

The Taylor impact test [3] was developed to evaluate the dynamic mechanical properties of materials. The methodology consists of a blunt cylinder shot perpendicularly ( 0 degrees NATO) against a smooth, rigid anvil. The recovered deformed sample is then analysed, and by applying an analytical relation, the dynamic yield stress of the material can be estimated. The investigation performed by Johnson [4] highlighted that the simplifications and assumptions at the base of the Taylor approach make the methodology not adequate for a precise estimation of the dynamic properties of materials, and more accurate experimental methodologies should be preferred. 
Taylor impact test have been used to assess deformation, fracture and damage evolution by Xiao et al. [5]. The influence of the hardness of the material on the deformation and fracture modes was assessed by shooting samples in the velocity range of $200-600 \mathrm{~m} / \mathrm{s}$. It was concluded that the samples experience five different fracture modes, depending on the hardness and the impact velocity: the softer specimens experience mushrooming, shear cracking and petalling while the harder projectiles experience mushrooming, shear cracking and fragmentation. The work was further implemented by Xiao et al. [6], confirming the previous conclusions.

The outcomes of the overdriven Taylor tests for Ferrocerium, recorded using a highspeed camera and described in detail in section 2, align with this description. Small plastic deformation and fragmentation were observed, offering essential information on the failure process and providing parameters used to evaluate numerical results.

The numerical analyses described in this work are modelled using Smoothed Particle Hydrodynamics (SPH) formulations available in the commercial software LS DYNA ${ }^{\circ}$. SPH is a meshless methodology suited for investigating phenomena involving very high strainrates and fracture. Even considering that this work focuses on the fragmentation of the material at relatively low velocities, the use of SPH is reasonable as the progression of the research project will investigate the material during high-velocity impacts.

\section{Experiment}

The samples used to perform the experiments were Ferrocerium cylindrical bars with a length of $40 \mathrm{~mm}$, a diameter of $8 \mathrm{~mm}(\mathrm{~L} / \mathrm{D}=5)$, and an initial mass of 13.2 grams. The specimens were shot against tungsten carbide anvils with initial velocities ranging between 60 and 140 $\mathrm{m} / \mathrm{s}$. Analysing the experimental outcomes, it was possible to observe a velocity threshold at $73 \mathrm{~m} / \mathrm{s}$ : for impact velocities up to this limit, the samples experienced small plastic deformation, while for velocities above the threshold, fragmentation and energy release were observed. Figure 1, for instance, depicts experiments performed with an impact velocity of $97.5 \mathrm{~m} / \mathrm{s}$. The frames show the IIER (top) and the fragmentation process (bottom) of two samples obtained at an impact velocity of $97.5 \mathrm{~m} / \mathrm{s}$.
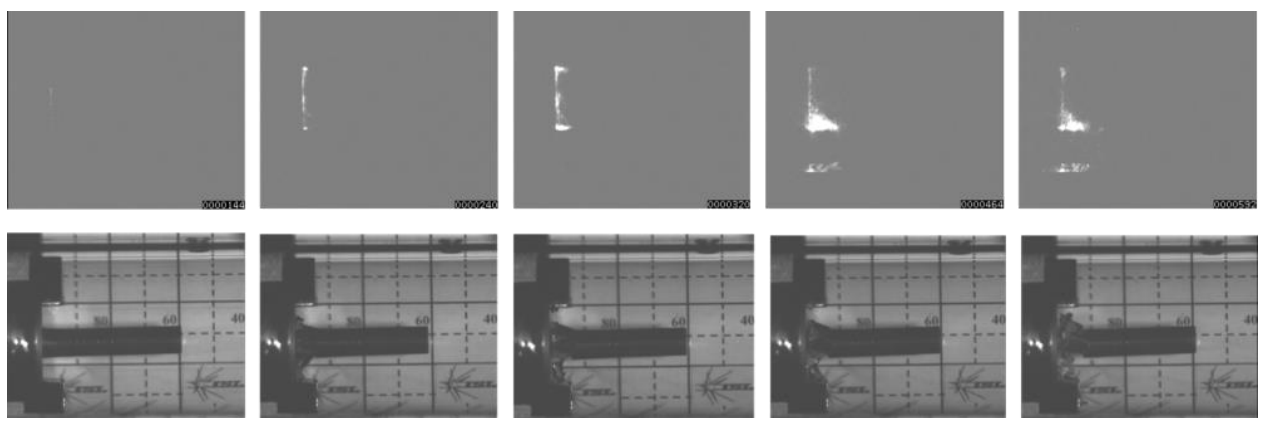

Fig. 1. Taylor tests: impact velocity of $97.5 \mathrm{~m} / \mathrm{s}$. Details of IIER (top) and fragmentation (bottom).

No reaction was recorded at impact velocities below the threshold, indicating a link between fragmentation and impact-induced energy release. The results of the experiments are summarised in Table 1. The decrease in the residual mass of the recovered samples is tied with the increase in the number of fragments. Furthermore, these preliminary results seem to indicate that the dimensions of the fragments decrease with increasing velocities, as indicated by the fragmented mass recovered. Interestingly, it was also observed that the total sum of the mass recovered was less than the initial mass when energy release occurred. Ongoing research is addressing the relationship between fracture and energy release. 
Table 1. Experimental outcomes: residual mass for samples at different impact velocities.

\begin{tabular}{|c|c|c|c|c|}
\hline SHOT & $\begin{array}{c}\text { Impact velocity } \\
(\mathbf{m} / \mathbf{s})\end{array}$ & $\begin{array}{c}\text { Sample residual } \\
\text { mass (g) }\end{array}$ & $\begin{array}{c}\text { Fragmented mass } \\
\text { recovered (g) }\end{array}$ & $\begin{array}{c}\text { Number of } \\
\text { fragments }\end{array}$ \\
\hline A & 73 & 13.2 & 0 & 0 \\
\hline B & 85 & 12.6 & 0.56 & 3 \\
\hline C & 86.6 & 11.4 & 0.45 & 6 \\
\hline D & 91 & 10.8 & 1.10 & 11 \\
\hline E & 97.5 & 10.6 & 1.81 & 8 \\
\hline F & 138.5 & 8.8 & 1.41 & 8 \\
\hline
\end{tabular}

\section{Numerical methods}

\subsection{Finite Element Method (FEM)}

The experiments performed by Xiao et al. [6] and discussed in section 1 were implemented with FEM analyses. The numerical results were in good agreement and replicated the fracture modes through the element erosion technique, observing, however, negative effects on the energy balances. Zhang et al. [7] assessed five different fracture models using experimental results as reference. The modified Johnson-Cook model, implemented with stress triaxiality cut-off, and the Cockcroft-Latham fracture criterion showed the best prediction capacities, replicating accurately shear cracking and fragmentation. Børvik et al. [8] and Rakvåg et al. [9] [10] performed a similar series of experiments, documenting the five main fracture modes also recorded by Xiao et al. [5] [6]. FEM numerical analyses accompanied the experimental studies. Besides, it was observed that a combination of fracture modes is highly possible.

\subsection{Smoothed Particle Hydrodynamics}

The SPH approach is a meshless Lagrangian numerical method developed by Lucy [11] and independently by Gingold and Monaghan [12] in 1977. The method has later been implemented for solid mechanics analyses and is now commonly used for the investigation of high strain rates phenomena. FEM is used in this work as verification and term of reference for the SPH results. In the progression of the research SPH will be used to model highvelocity impact: SPH is more efficient as the time step stays constant during the analyses, as described in expamples in [13].

For the traditional SPH formulation, the kernel functions are computed based on the instantaneous current particle positions. For this formulation, referred to as Eulerian kernel function, particles can move inside and out of the influence domain of the kernel, leading to tensile instability, as observed by Swegle [14]. In order to overcome the tensile instability, Total Lagrangian SPH formulation, which calculates kernel functions referring to the reference configuration, was developed [15] [16].

Zhou et al. [17] tried to apply the SPH methodology to Taylor impact tests, obtaining first encouraging results. In the study, the fracture experienced by the projectile in the numerical result was referred to as "numerical fracture" due to the loss of connection between particles. This subject has also been discussed by Xu et al. [18], highlighting that the fracture experienced in SPH results may be affected by the numerical instability present in SPH. In section 4.1, the effects of kernel formulation and tensile instability observed on numerical results are briefly described.

One of the most common constitutive models for high-velocity impacts is the JohnsonCook model [19], which describes the flow stress as a function of strain, strain rate and temperature effects. In the present work, the modified Johnson-Cook constitutive model was chosen as it allowed using the Cockcroft-Latham failure criterion (CL), which is an energy- 
based fracture model that depends on a single parameter [20]. The CL does not take into account microscopic mechanical aspects as it is phenomenological. Though, it has been applied successfully in simulations involving impacts and brittle fracture.

\section{Results and discussion}

The experimental result obtained from test A $(73 \mathrm{~m} / \mathrm{s})$ showed small plastic deformation and no specimen failure. The outcome was used to compare different formulations and discretisation of the models. In this first approach, a rigid wall represents the target. The failure of the samples observed at higher velocities have been analysed numerically with a TLSPH model.

The FEM model uses 153600 hexahedral fully integrated elements with a resolution of $0.25 \mathrm{~mm}$, while the TLSPH model uses 259000 particles with a resolution of $0.2 \mathrm{~mm}$. Furthermore, the FEM model uses a butterfly mesh, adequate for simulating this application, while the particle distribution for the SPH models was generated using the algorithm developed by Becker et al. [21], as shown in Figure 2. The particle distribution algorithm has already been applied in ballistic problems, proving the effectiveness in avoiding numerical fracture [22].
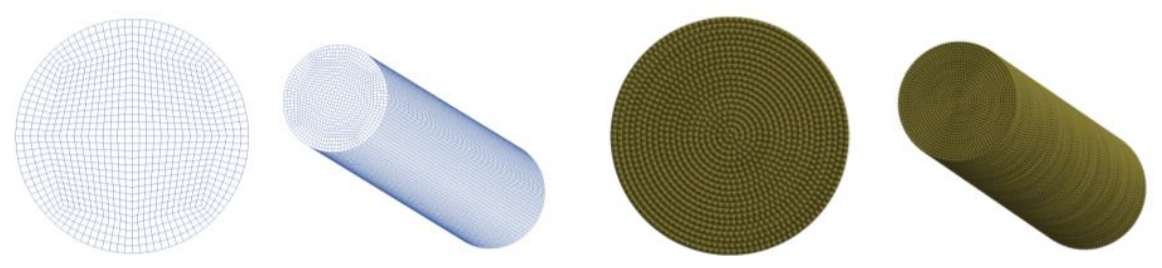

Fig. 2. FEM meshed sample (left) and SPH sample (right).

\subsection{Formulation, accuracy and stability analysis}

Figure 3 shows the comparison between numerical results obtained using a formulation with re-normalised Eulerian kernel and a total Lagrangian formulation (TLSPH), respectively shown as a red and a blue curve, at an impact velocity of $73 \mathrm{~m} / \mathrm{s}$. The red curve clearly shows the effects of tensile instability on the length variation (left), visible as numerical dissipation of wave amplitude and period.
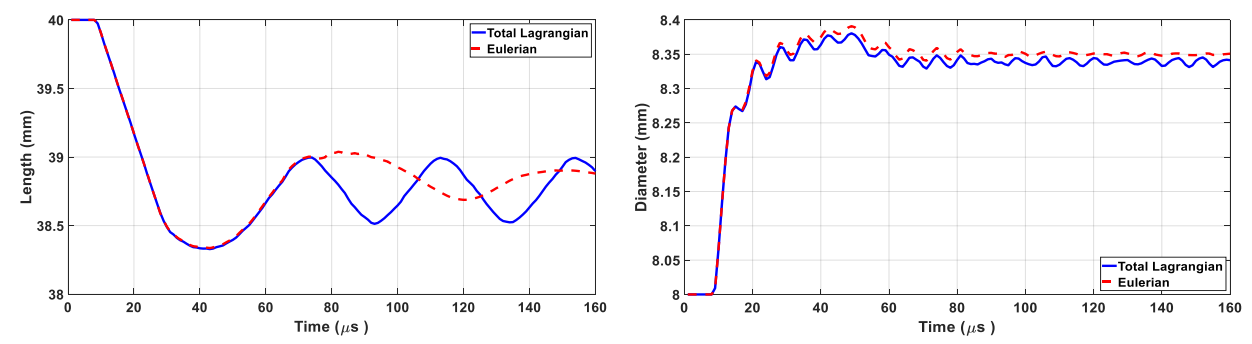

Fig. 3. Numerical results showing plastic deformation comparison between re-normalised Eulerian SPH (red) and TLSPH (blue) formulations.

The plastic deformation, shown in terms of length and diameter variations, is also depicted in Figure 4. The TLSPH measurements are based on the outer dimension of the samples, i.e. the measured distance between the centre of the particles plus a delta representing the outer diameter. The graph on the right compares the variation of the diameter for the impact surface, expressed in percentage. A difference of $1 \%$ in the final configuration is measurable between the FEM (yellow curve) and TLSPH results obtained using an increasing number of particles. 

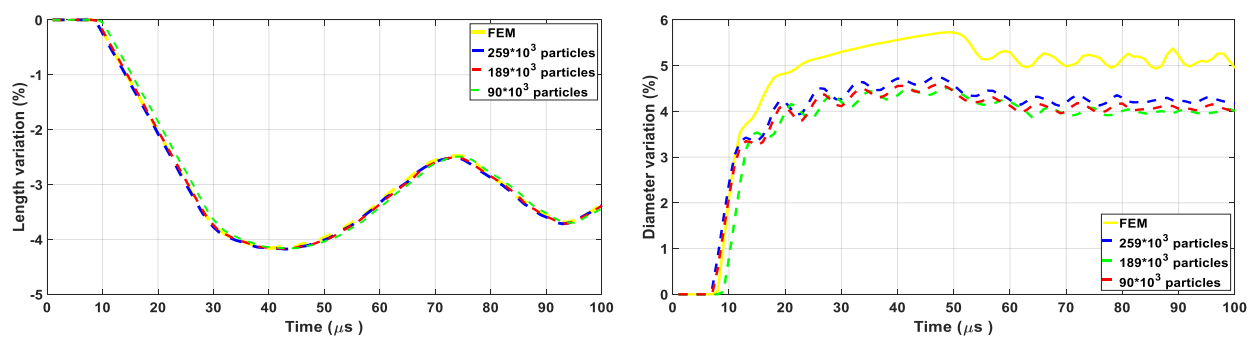

Fig. 4. Numerical variation in length (left) and diameter (right) obtained from FEM and TLSPH simulations at impact velocity of $73 \mathrm{~m} / \mathrm{s}$.

The graph on the left in Figure 4 indicates the length variation curves, also expressed in percentage. In this graph, the FEM and the different TLSPH curves are equivalent, independently from the number of particles used: the shortening of the specimen during the impact, followed by a vibration phase for all the analyses performed is well captured.

The plots depicted in Figure 5 show the comparison between the FEM and TLSPH (259000 particles) results for Von Mises stress, pressure, and uniaxial stress, measured at $7.75 \mathrm{~mm}$ (left) and $12.2 \mathrm{~mm}$ (right) from the impact surface. The solutions show very similar behaviour. The minor differences are linked with the slightly different discretisation refinement and the nature of the elements/formulation used. Overall, the TLSPH model shows accuracy and stability.
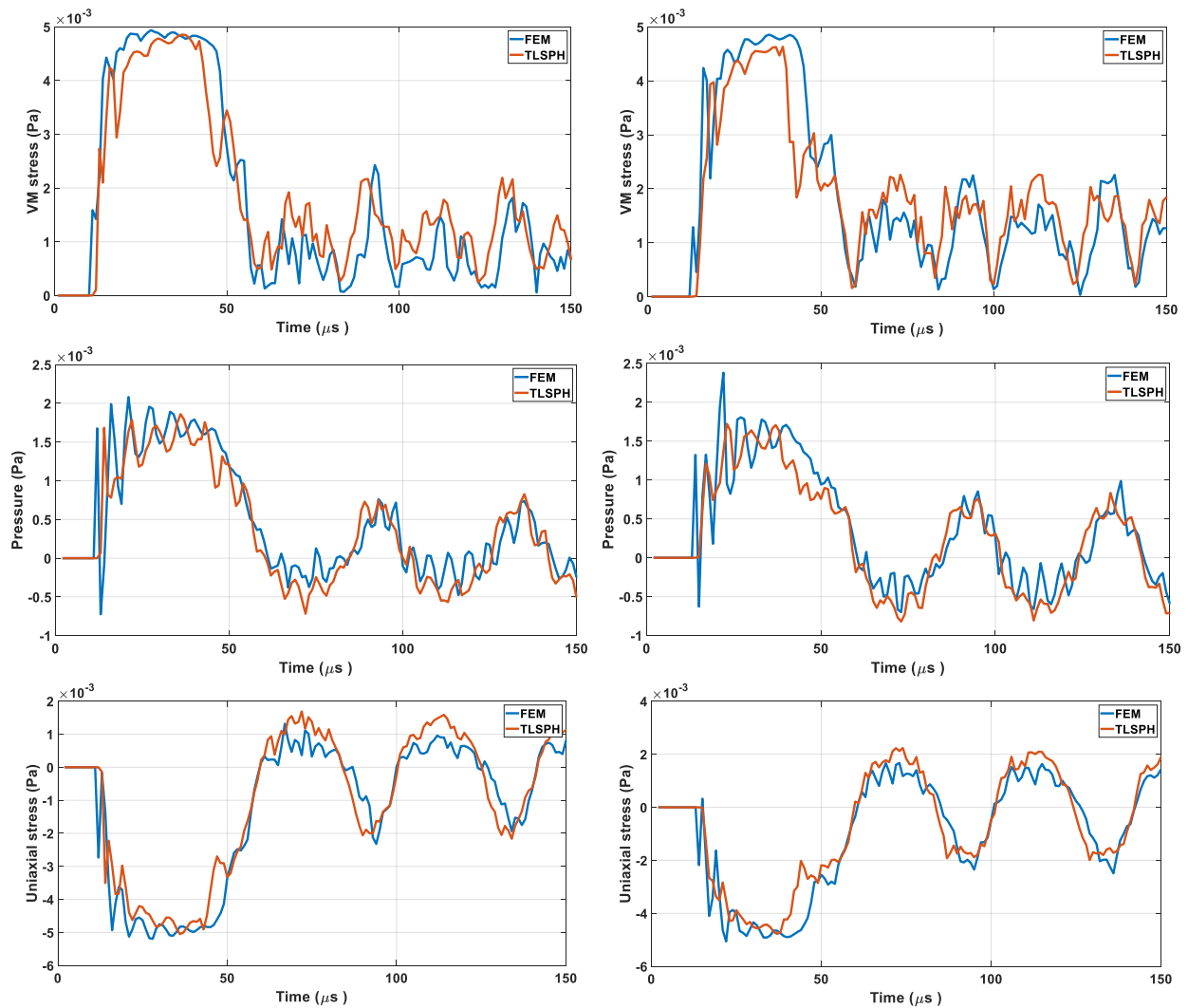

Fig. 5. Comparison of numerical Von Mises stress, pressure and uniaxial stress evolutions for FEM (blue curve) and TLSPH (red curve) at 7.75 and $12.2 \mathrm{~mm}$ from the impact surface; impact velocity of $73 \mathrm{~m} / \mathrm{s}$. 


\subsection{Fragmentation analysis}

The experiments performed showed the fragmentation of the samples for impact velocities higher than $73 \mathrm{~m} / \mathrm{s}$. The residual masses of the samples were used as parameter for the evaluation of numerical investigations, summarised in Table 2, showing first interesting results. Figure 6 displays a visual qualitative comparison of numerical outcomes for the Taylor test performed at $97.5 \mathrm{~m} / \mathrm{s}$ using a FEM model and $\mathrm{SPH}$ with two different kernel formulations. The FEM results are mesh dependent, and the sensitivity analysis is a critical step to consider while using the methodology. Furthermore, to avoid symmetry in the final result, a perturbation in the node distribution has been applied. The SPH results predict fracture and fragmentation accurately.

Formulation

a)

b) Eulerian

c) TL SPH.
Side view

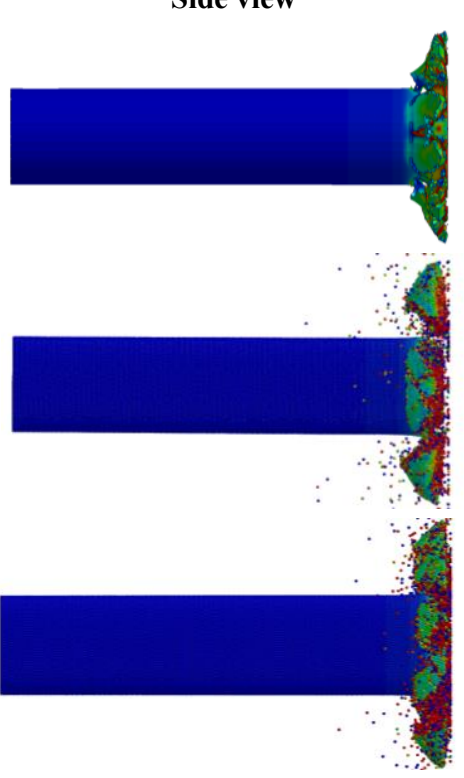

Top view
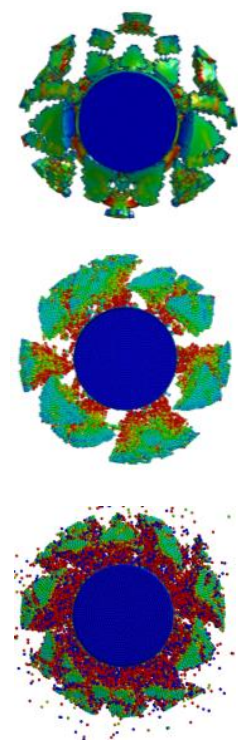

Fig. 6. Visual result of fragmentation obtained using CL fracture criterion. Impact velocity $97.5 \mathrm{~m} / \mathrm{s}$.

Eulerian kernel formulations can be affected by tensile instability, manifesting with clustering of particles [23] and artificial voids [24]. The result shown in Figure $5 \mathrm{~b}$ has been tested for numerical fracture by repeating the simulation without including the failure criterion, proving that the criterion triggers the fracture.

Table 2. Comparison of experimental and numerical residual masses at different impact velocities.

\begin{tabular}{|c|c|c|c|c|}
\hline SHOT & $\mathbf{V}_{\mathbf{i}}(\mathbf{m} / \mathbf{s})$ & $\begin{array}{c}\text { Experimental Residual } \\
\text { mass } \mathbf{( g )}\end{array}$ & $\begin{array}{c}\text { Numerical Residual } \\
\text { mass (g) }\end{array}$ & Error (\%) \\
\hline $\mathbf{C}$ & 86.6 & 11.4 & 11.5 & +2.56 \\
\hline $\mathbf{D}$ & 91 & 10.8 & 10.8 & 0 \\
\hline $\mathbf{E}$ & 97.5 & 10.6 & 10.3 & -2.83 \\
\hline $\mathbf{N 1}$ & 110 & - & 9.85 & - \\
\hline $\mathbf{N 2}$ & 120 & - & 9.41 & - \\
\hline $\mathbf{N 3}$ & 130 & - & 8.96 & - \\
\hline $\mathbf{F}$ & 138.5 & 8.8 & 7.98 & $-9.32 \%$ \\
\hline $\mathbf{N} 4$ & 150 & - & 6.26 & - \\
\hline
\end{tabular}


Table 2 lists the residual masses of the main samples recovered from the experiments and compares them with the TLSPH numerical results, indicating sufficient accuracy in the final mass evaluations and representing a good starting point for implementing the model. The data indicate that the accuracy of the prediction decreases, and the error becomes more significant at higher velocities, as can be also observed in Figure 7. The graph displays the comparison between experimental and numerical residual masses. The curve fit, shown in red, is obtained from the experimental outcomes. The numerical results are in line with the experimental outcomes with error in the range of less than $3 \%$ for shot $\mathrm{B}, \mathrm{C}$ and $\mathrm{D}$, while for shot $\mathrm{E}$, the error becomes significant at almost $10 \%$, indicating the need for a more accurate model. Further investigation will be performed substituting the rigidwall representing the target as a set of particles.

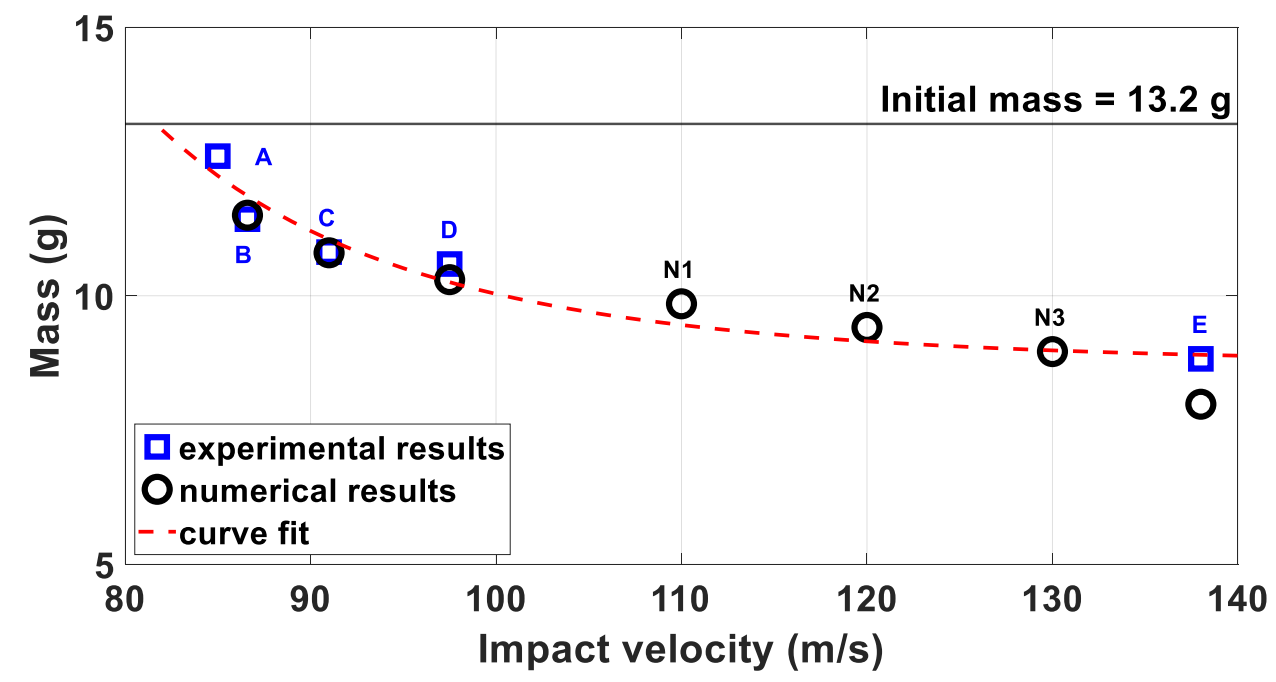

Fig. 7. Comparison between numerical and experimental results in terms of residual masses of samples at different impact velocities. The curve fit, displayed in red, was obtained from the experimental data.

\section{Conclusions}

The Taylor impact tests were used to investigate fracture for Ferrocerium samples. Brittle fracture and impact-induced energy release were observed for impact velocities in the range of $73 \mathrm{~m} / \mathrm{s}$ to $140 \mathrm{~m} / \mathrm{s}$.

The Total Lagrangian SPH model was proven to be adequate in reproducing the small plastic deformation experienced at lower velocities by the samples. The computed final diameter and length were compared with the experimental results, showing an error smaller than $2 \%$. This case was also used to highlight how tensile instability affects numerical results: the conventional Eulerian formulation suffers from tensile instability, clearly observable in the deformation plots as "numerical dissipation".

The model can capture and predict the final weights of the samples within $10 \%$ of the experimental observations. This preliminary setup described in this work proves to be sufficiently adequate for the description of the inert fragmentation. It represents the foundation for additional investigations on fracture, impact-induced energy release, and the implementation of more accurate models. 


\section{References}

1. D. L. Hastings and E. L. Dreizin, Adv. Eng. Mater. , 20, (2017).

2. K. Reinhardt and H. Winkler, Ullmann's Encyclopedia of Industrial Chemistry, (2000).

3. G. I. Taylor, Proc. R. Soc. Lond. A, 194, (1948).

4. G. R. Johnson, T. J. Holmquist, J. Appl. Phys., 64, (1988).

5. X. Xiao, W. Zhang, G. Wei and Z. Mu, Mater. Des., 31, (2010).

6. X. Xiao, W. Zhang, G. Wei, Z. Mu and Z. Guo, Mater. Des., 32, (2011).

7. W. Zhang, X. Xiao, G. Wei and Z. Guo, AIP conference proceedings 1426, (2012).

8. T. Børvik, O. S. Hopperstad, M. Langseth and K. A. Malo, Int. J. Impact Eng., 28, no. 4, (2003).

9. K. G. Rakvåg, T. Børvik, O. S. Hopperstad and I. Westermann, EPJ Web of Conferences, vol. 26, (2012).

10. K. G. Rakvåg, T. Børvik and O. S. Hopperstad, Int. J. Solids Struct., 51, (2014).

11. L. B. Lucy, Astronomical Journal, 82, (1977).

12. R. A. Gingold and J. J. Monaghan, Monthly Notices of the Royal Astronomical Society, (1977).

13. P. W. Cleary, R. Das, IUTAM Symposium on Theoretical, Modelling and Computational Aspects of Inelastic Media, (2008).

14. J. Swegle, D. Hicks, S. Attaway, Journal of computational physics, 116 (1), (1995).

15. R. Vignjevic, JR Reveles, J. Campbell, Comput Model Eng Sci, 14 (3), (2006).

16. T. De Vuyst, R. Vignjevic, International Journal of Fracture, 180, (2013).

17. C. E. Zhou, G. R. Liu and X. Han, Computational Methods, Springer, (2006).

18. F. Xu, Y. Zhao, Y. Li and M. Kikuchi, Acta Mechanica Solida Sinica, 23, no. 1, (2010).

19. G. R. Johnson, W. H. Cook, Proceedings of the 7th International Symposium on Ballistics, (1983).

20. LS-DYNA manual.

21. M. Becker, M Seidl, M. Mehl, M. Souli, Proceedings of the 2019 Hypervelocity Impact Symposium, (2019).

22. M. Becker, M. Seidl, M. Mehl, M. Souli, J. F. Legendre, $12^{\text {th }}$ European LS-DYNA Conference, (2019).

23. J. J. Monaghan, Journal of Computational Physics, 159, (2000).

24. V. Mehra, S. CD, V. Mishra, S. Chaturvedi, Journal of Physics: Conference Series, 377, (2012). 